\title{
The Research Center for the History of Science and Technology Inaugurated at Nankai University
}

\section{LÜXin 吕昕, YU Yueyuan 俞月圆}

(Institute for the History of Natural Sciences, Chinese Academy of Sciences, Beijing 100190, China)

$\mathrm{T}$ The day June 23, 2021 bore witness to the inauguration of the Research Center for the History of Science and Technology (RCHST) of Nankai University (NKU) and the signing ceremony of a cooperation agreement between the Institute for the History of Natural Sciences (IHNS) of the Chinese Academy of Sciences and NKU in Tianjin (Figure 1). The attendees included scientists, historians, and students from NKU, IHNS, Peking University (PKU), Tsinghua University (THU), University of Science and Technology of China (USTC), and other institutions. The celebration of this commemorative moment was accompanied by congratulatory letters from numerous counterparts, such as the Max Planck Institute for the History of Science, the S. I. Vavilov Institute for the History of Science and Technology of the Russian Academy of Sciences, Technische Universität Berlin, Université de Paris, Shanghai Jiao Tong University, USTC, and Inner Mongolia Normal University.

During the inauguration, Academician Cao Xuetao 曹雪涛, president of NKU, highlighted the profound implications of the joint founding of the RCHST by NKU and IHNS. First and foremost, it will complete the structure of the discipline of history at NKU and provide a high-level platform on which the discipline of history of science and technology (HST) can move forward. Second, it will help to bridge the gap between the humanities and sciences, empowering students with interdisciplinary knowledge structures and a more comprehensive view of history. Last but not least, it will enable the forging of dialogue and collaboration between scholars in the fields of science, technology, and culture.

Prof. Guan Xiaowu 关晓武, deputy director of the IHNS, underlined that NKU has traditionally been a leader in the discipline of history. He extended the hope that

This piece of news was copyedited by John Moffett. 


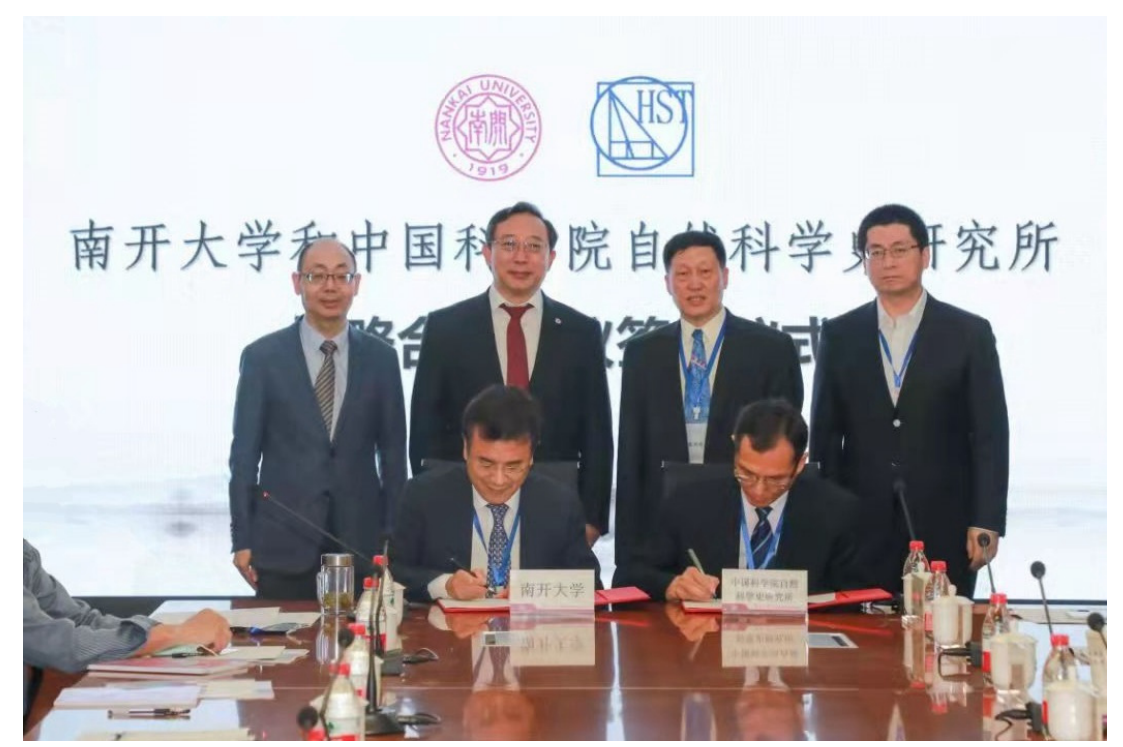

Figure 1: Academician Chen Jun 陈军, vice president of NKU, and Prof. Guan Xiaowu 关晓武, deputy director of the IHNS, signed the cooperation agreement.

NKU and the IHNS will capitalize upon their academic specialties, and proactively cooperate in discipline construction, academic research, and student nurturing. In doing so, their collective endeavors will elevate research on HST to new heights and open up new research fields, thus ushering in a new phase for the discipline in China. Prof. Zhang Baichun 张柏春, director of the NKU RCHST, stated that HST would enrich NKU's research landscape, which already encompassed Chinese history, world history, and archaeology. The research center would carve out new fields based on established disciplines, such as social history of medicine, environmental history, history of astronomy, and history of mechanics and technology. Domestic and international exchange and cooperation would also be actively pursued.

The inauguration of the RCHST brings NKU into the group of thirteen universities, apart from the IHNS, that train doctoral students in HST and history of medicine on the Chinese mainland. Most of these universities have developed HST as a separate science discipline, independent of the department (or faculty) of history. The RCHST at Nankai University, underpinned by the Faculty of History, is committed to further integrating HST with such branches of history as world history and Chinese history, thus conducting collaborative research on complex issues at the intersection of science, technology, and society. What adds further to the research landscape of history is the alliance between historians of S\&T and scientists in China, as exemplified by heads of multiple NKU schools and colleges who staunchly support collaborative research on HST, including the School of Medicine, College of Life Sciences, College of Environmental Science and Engineering, School of Physics, and College of Computer Science. 